\title{
Metodología para la evaluación interna de una cadena de valor
}

\author{
Methodology for the internal assessment of a value chain
}

\author{
Eduardo Felipe Vásquez-Barajas ${ }^{1}$, Liliana Marcela Bastos-Osorio ${ }^{2}$ y Johanna Milena Mogrovejo-Andrade M $^{3}$ \\ ${ }^{1}$ Universidad Francisco de Paula Santander, Colombia. Email: eduardofelipevb@ufps.edu.co \\ ${ }^{2}$ Universidad Francisco de Paula Santander, Colombia. Email: lilianamarcelabo@ufps.edu.co \\ ${ }^{3}$ Universidad Francisco de Paula Santander, Colombia. Email: johannamogrovejo@ufps.edu.co
}

Para citar este artículo: Vásquez, E., Bastos, L. y Mogrovejo, J. (2018). Metodología para la evaluación interna de una cadena de valor. Clío América, 14(27), 401-408. http://dx.doi.org/10.21676/23897848.3685

Recibido: 26 julio de 2019

Aceptado: 30 de enero de 2020

Publicado en línea: marzo 03 de 2020

\section{Palabras clave: cadena de valor; competitividad ; creación de valor; evaluación; metodología.}

JEL: M100.

Keywords: value chain; competitiven ess; value creation; assessment; methodology

\section{RESUMEN}

El objetivo de este artículo es diseñar una metodología para la evaluación interna de una cadena de valor. La metodología comprende un tipo de investigación cuantitativo, un método de investigación deductivo y un tipo de estudio explicativo. Sobre los resultados, la metodología diseñada para la evaluación interna se enfoca en el esquema de cadena de valor de Michael Porter con sus actividades principales y actividades de apoyo cruzadas en una matriz que permite describir y ponderar la relación de cada actividad principal con cada actividad de apoyo. La matriz propuesta ubica las 5 actividades principales en el eje vertical y las 4 actividades de apoyo en el eje horizontal; distribuye el $100 \%$ entre las 9 actividades de la siguiente manera: un porcentaje superior al $50 \%$ para las 5 actividades principales por ser la parte central de una cadena de valor, y el porcentaje restante para las actividades de apoyo porque sin ellas las actividades principales no estarían soportadas. En conclusión, el diseño de la metodología propuesta para la evaluación interna de la cadena de valor permite identificar actividades principales y de apoyo fuertes como ventajas competitivas o por mejorar como falencias, así como relaciones entre ellas.

\section{ABSTRACT}

The objective of this paper is to design a methodology for the internal assessment of a value chain. The methodology includes a type of quantitative research, a deductive research method and a type of explanatory study. Regarding the results, the methodology designed for the internal assessment focuses on Michael Porter's value chain scheme with its main and support activities in a matrix that allows describing and weighing the relationship of each main activity with each support activity. The proposed matrix locates the 5 main activities on the vertical axis and the 4 support activities on the horizontal axis. It distributes $100 \%$ among the 9 activities as follows: a percentage higher than $50 \%$ for the 5 main activities because they are the central part of a value chain. The remaining percentage for the support activities since, without them, the main activities would not have support. In conclusion, the design of the proposed methodology for the internal assessment of the value chain makes it possible to identify strong main and support activities as competitiveness advantages or to be improved as weaknesses, as the relations between them. 


\section{METODOLOGÍA PARA LA EVALUACIÓN INTERNA DE UNA CADENA DE VALOR}

\section{INTRODUCCIÓN}

Según Porter (2006), la cadena de valor es una serie de actividades de una empresa o sector que desarrolla un producto para ofrecer al consumidor o cliente final. Dicha cadena facilita la creación de alianzas entre los actores, permitiendo un uso más eficiente de los recursos, facilitando el flujo de información entre los actores y generando soluciones de manera conjunta a problemas y cuellos de botella. Además, Porter (2006) indica que la cadena de valor consiste en dividir la organización en sus actividades estratégicas (primarias y de apoyo), con el fin de entender la composición y la afectación de los costos, así como las actividades actuales y potenciales que generen diferenciación, logrando ventajas competitivas.

El control de estos costos y la generación de valor agregado en las actividades de la cadena es crucial para lograr y mantener la ventaja competitiva y los beneficios a corto y largo plazo (Shapiro et al., 1993). De acuerdo con (Porter, 2006, como se citó en Devoto, 2011), las actividades de valor se dividen en dos grupos expuestos a continuación: actividades primarias y actividades de apoyo.

Actividades primarias: logística de entrada, operaciones, logística de salida, marketing y ventas, servicio postventa.

Actividades de apoyo: infraestructura de gestión, manejo de recursos humanos, desarrollo tecnológico y adquisiciones.

Además, (Porter, 2006, como se citó en Devoto, 2011) plantea que las actividades primarias y de apoyo pueden subclasificarse como actividades directas e indirectas respectivamente, así como la relación con actividades llamadas seguros de calidad como el monitoreo y el control de calidad.

Porter (1985) enfatiza en la importancia de enfocar los eslabones entre las actividades en la cadena de valor. Los eslabones llevan a la ventaja competitiva de dos formas: optimización y coordinación. Estos frecuentemente indican intercambios entre actividades, por lo que una organización debe optimizar los eslabones reflejando su estrategia con el fin de lograr ventajas competitivas. La habilidad para coordinar los eslabones frecuentemente reduce los costos o potencia la diferenciación (Shapiro et al., 1993).

La cadena de valor hace parte de un sistema de valor que inicia en los proveedores de los proveedores y llega a los clientes de los clientes. De esa forma se van creando eslabones y se va definiendo la dinámica de las cadenas. Por ejemplo, "el fabricante tiene la función de la calidad y la innovación en el producto, el mayorista se encarga de la consolidación y distribución indicada de los productos, el detallista de la comercialización del producto y así sucesivamente" (Quintero y Sánchez, 2006). De acuerdo con Chávez (2012), la cadena de valor hace más fácil la generación de alianzas para la productividad, lo que lleva a una utilización o empleo de recursos de manera más eficiente, a exaltar las actividades de distribución y el mercadeo como variables estratégicas para un incremento en la competitividad, a mejorar el intercambio de información entre los diversos actores, y a ayudar a la generación de soluciones de forma sistemática junto con el diagnóstico y la definición de diversos problemas y cuellos de botella a través de la cadena integral. Por ende, es necesario analizar cada eslabón de la cadena.

Siendo así, dentro de la cadena de valor también se deben considerar los eslabones, dado que también son una fuente de ventaja competitiva. Los eslabones, según (Porter 2006, como se citó en Devoto, 2011), pueden ser:

Interiores: reflejan la interdependencia entre las distintas actividades de la cadena de valor. Los más obvios son aquellos existentes entre las actividades primarias y las de apoyo, representados por las líneas segmentadas en la cadena de valor.

Verticales: se encuentran entre la cadena de valor de la empresa y las cadenas de valor de los proveedores y de los distribuidores.

El diagnóstico de la cadena de valor es una herramienta para desglosar una empresa o sector en actividades estratégicamente importantes y entender su impacto en el comportamiento de los costos y la diferenciación. Las interdependencias proveen oportunidades para la optimización y la resolución de problemas de coordinación entre actividades dentro de la cadena, con las cadenas de valor tanto de los encargados del suministro como la de los clientes, así como con las cadenas de valor de otras unidades de negocios estratégicas dentro del mismo segmento (Hergert y Morris, 1989). Como un marco para la planeación estratégica, el análisis de cadena de valor tiene características distintivas, las cuales son: un énfasis en identificar la fuente de ventaja competitiva sostenible; una insistencia en la importancia de eslabones complejos y sus interrelaciones; y la identificación de estrategias genéricas en las diversas actividades de creación de valor (Hergert y Morris, 1989). 
Así mismo, un análisis estratégico de las cadenas de valor involucra el suministro de los proveedores o la logística de entrada para desarrollar bienes específicos desde bienes básicos como los commodities (Craig y Douglas, 1997). Además, Wright (1987) señala que el diagnóstico de la cadena de valor debe comprender las actividades principales que van desde las compras específicas para proveerse, hasta la terminación de los bienes y la actividad de marketing y seguimiento al cliente.

Siguiendo con lo anterior, Piana et al. (2005) indican que las actividades principales de una cadena de valor comprenden la actividad del suministro con especificaciones estrictas de materias primas; sigue con la actividad de operaciones en la que se hace relación a la vida útil del producto, el confort para el consumidor y la apariencia mejorada del producto; luego está la actividad de distribución con la pronta entrega del producto terminado y su manejo manteniendo las condiciones ambientales; después la actividad de ventas y mercadeo que se basa en los atributos del producto y en el cumplimiento al consumidor; y por último la actividad de promoción y seguimiento que trata sobre la retroalimentación y ampliación de la línea de producto.

Inclusive, las cadenas de valor pueden afectarse por productos terminados con bajo valor creado (Boehlje, 1999); por una reducción en los márgenes de retorno; por una red de miles de participantes potenciales más que un conjunto integrado de negocios; por variables sociales fuertes como tendencias de consumo o conservación de la naturaleza, así como por objetivos económicos (Valentine, 2005). Los consumidores también tienen una influencia en la cadena de valor a través de retroalimentaciones positivas o negativas a través de las preferencias o estilos de vida (Perona y Miragliotta, 2004).

Además, las cadenas de valor con enfoque agrícola son fundamentales para el crecimiento o el mantenimiento de las economías regionales y nacionales, y la riqueza y bienestar de los productores. El suministro sostenible, principalmente en un ambiente incierto y dinámico, es un mayor desafío; inclusive, el cambio climático, la degradación de recursos naturales, las exigencias del mercado y los mercados globales ejercen presión sobre las cadenas de valor agrícolas (Higgins et al., 2010).

\section{METODOLOGÍA}

La metodología para la investigación respectiva comprendió una investigación cuantitativa con un método aplicado de deducción y un estudio de índole explicativo. Así mismo, se consultaron los aportes de diferentes autores sobre la cadena de valor y los aportes de autores para el análisis o evaluación de la cadena de valor. El diseño de la metodología de evaluación interna de una cadena de valor propuesta por los investigadores autores del presente artículo tomó como referente teórico la competitividad en la cadena de valor propuesta por Michael Porter y tomó en cuenta las actividades principales y las actividades de apoyo, para luego, a través de una serie de criterios establecidos por los autores, se realizará la evaluación. Antes de haber iniciado el diseño de una metodología de evaluación interna para una cadena de valor, se revisaron como antecedentes tres metodologías de otros autores sobre la evaluación de las cadenas de valor: metodología de evaluación de acuerdo a factores de integración, según De la Hoz et al. (2011); metodología de evaluación por análisis (Fortalezas, Oportunidades, Debilidades y Amenazas - FODA) y ponderación de actividades en categorías, de acuerdo con Arce y Calves (2008); y metodología de evaluación y priorización de cadenas de valor agrícolas para la responsabilidad social empresarial, según Scott (2014). Luego de revisar las metodologías propuestas por otros autores, se procedió a diseñar una nueva metodología para evaluar internamente una cadena de valor con la finalidad de lograr encontrar o identificar las actividades principales y las actividades de apoyo más fuertes y débiles que representaran ventajas competitivas y factores o variables a mejorar, considerando los resultados para la toma de decisiones relacionada con el aumento de la productividad, la competitividad y la internacionalización a futuro (Vásquez y Bastos, 2018).

La metodología de evaluación interna para una cadena de valor buscó evaluar cuantitativa y cualitativamente las actividades principales y de apoyo de una cadena de valor. La evaluación interna fue cuantitativa, dado que las actividades de la cadena de valor fueron medidas a través de escalas y ponderaciones que permitieron identificar ventajas competitivas y/o debilidades; al mismo tiempo fue cualitativa por las descripciones de las relaciones entre cada actividad de apoyo con cada actividad principal que pudieran llevar a identificar eslabones de una mejor manera. Cabe aclarar que la presente metodología fue validada por expertos académicos en cadenas de valor, competitividad e innovación.

\section{RESULTADOS}

La evaluación interna se enfocó en el esquema de la cadena de valor de Porter con sus actividades principales y actividades de apoyo cruzadas en una matriz (Tabla 1) que permite describir y ponderar la relación de cada actividad principal con cada actividad de apoyo. En el diseño de esta matriz se tomó en cuenta el modelo y la metodología de 
matriz propuesto por Pérez y Calves (2008), así como a Arce y Calves (2008). En ella se pondera de cero a cinco la relación de actividad de apoyo contra cada actividad principal y también se toma en cuenta el modelo y la metodología de matriz de preselección de mercados o países (usualmente trabajada en planes de internacionalización y ampliamente replicada por Procolombia). En dicha matriz se asigna un porcentaje a cada variable hasta completar el $100 \%$ y luego se califica con una escala que se multiplica con el porcentaje asignado para obtener un valor total.

La matriz propuesta ubica las cinco actividades principales en el eje vertical y las cuatro actividades de apoyo en el eje horizontal, distribuyendo el $100 \%$ entre las nueve actividades de la siguiente manera: $60 \%$ para las cinco actividades principales porque, como su nombre lo indica, son la parte central de la cadena; y el $40 \%$ para las actividades de apoyo, porque sin ellas las actividades principales no estarían soportadas y no se podrían desarrollar. El $60 \%$ de las actividades principales se decide distribuir de manera equitativa entre las cinco actividades, es decir, $12 \%$ para cada una porque se le da una misma importancia a la logística de entrada (proveeduría), las operaciones (producción), la logística de salida (distribución), el marketing y las ventas (comercialización), y el seguimiento al cliente (postventa); así mismo, el $40 \%$ de las actividades de apoyo se dividen de manera igualitaria por la misma razón entre las cuatro actividades, es decir, $10 \%$ para cada una de estas actividades, las cuales son infraestructura (organizacional), manejo de recursos humanos, desarrollo tecnológico o técnico, y adquisiciones (de soporte). Cabe aclarar que el evaluador puede asignar cada uno de estos porcentajes según su criterio sin exceder el total del $100 \%$ entre todas las actividades de la cadena de valor.

Tabla 1. Metodología para evaluación interna de la cadena de valor

\begin{tabular}{|c|c|c|c|c|c|c|c|c|c|c|c|c|c|c|}
\hline \multirow{3}{*}{\multicolumn{2}{|c|}{\begin{tabular}{|c|}
$\begin{array}{c}\text { Matriz para } \\
\text { evaluar } \\
\text { cadena de valor }\end{array}$ \\
Actividades \\
Principales \\
\end{tabular}}} & \multicolumn{13}{|c|}{ Actividades de Soporte } \\
\hline & & \multicolumn{3}{|c|}{$\begin{array}{c}\text { Infraestructura } \\
\text { Organizacional }\end{array}$} & \multicolumn{3}{|c|}{$\begin{array}{c}\text { Manejo de } \\
\text { Recursos Humanos }\end{array}$} & \multicolumn{3}{|c|}{$\begin{array}{c}\text { Desarrollo } \\
\text { Tecnológico }\end{array}$} & \multicolumn{3}{|c|}{ Adquisiciones } & \multirow{2}{*}{$\begin{array}{l}\text { Total } \\
40 \% \\
\end{array}$} \\
\hline & & Descrip. & $\begin{array}{l}10 \\
\%\end{array}$ & $\mathbf{C}$ & Descrip. & $10 \%$ & $\mathbf{C}$ & Descrip. & $\begin{array}{l}10 \\
\%\end{array}$ & $\mathbf{C}$ & \begin{tabular}{c|} 
Descrip \\
.
\end{tabular} & $10 \%$ & $\mathbf{C}$ & \\
\hline $\begin{array}{c}\text { Logística } \\
\text { de Entrada }\end{array}$ & $12 \%$ & & & & & & & & & & & & & \\
\hline $\begin{array}{c}\text { Operacione } \\
\text { s }\end{array}$ & $12 \%$ & & & & & & & & & & & & & \\
\hline $\begin{array}{l}\text { Logística } \\
\text { de Salida }\end{array}$ & $12 \%$ & & & & & & & & & & & & & \\
\hline $\begin{array}{c}\text { Marketing } \\
\text { y Ventas }\end{array}$ & $12 \%$ & & & & & & & & & & & & & \\
\hline $\begin{array}{l}\text { Seguimient } \\
\text { o al Cliente }\end{array}$ & $12 \%$ & & & & & & & & & & & & & \\
\hline Total & $60 \%$ & & & & & & & & & & & & & Margen \\
\hline
\end{tabular}

Fuente: elaboración propia

Luego se procedió a describir la relación e identificar las fortalezas y debilidades entre cada actividad de apoyo contra cada actividad principal (de acuerdo con la información obtenida en el diseño de la cadena de valor). Esta descripción fue concisa y no muy extensa.

A continuación, se calificó con una escala de Likert de 1 a 5 (Tabla 2) donde 5 indica que hay un eslabón fuerte generando valor que se convierte en una ventaja competitiva identificada, 4 indica un potencial de ventaja competitiva en el eslabón, 3 indica un nivel regular o medio donde no hay ni ventaja competitiva ni debilidad (pero sí susceptible de mejora), 2 indica que hay una debilidad menor, mientras que 1 indica que no hay un eslabón y representa una debilidad mayor. Así mismo, esta escala de calificación numérica vino acompañada de colores para facilitar la identificación de las calificaciones en la matriz. 
Tabla 2. Escala de calificación de actividades

\begin{tabular}{|c|c|c|}
\hline NÚMERO & COLOR & INTERPRETACIÓN \\
\hline $\mathbf{5}$ & Verde & Ventaja competitiva \\
\hline $\mathbf{4}$ & Azul & Potencial de ventaja competitiva \\
\hline $\mathbf{3}$ & Amarillo & Regular \\
\hline $\mathbf{2}$ & Naranja & Debilidad menor \\
\hline $\mathbf{1}$ & Rojo & Debilidad mayor \\
\hline
\end{tabular}

Fuente: elaboración propia

La calificación se hizo según la interpretación y el criterio del evaluador de acuerdo con el análisis realizado y la relación que encontró entre cada actividad de apoyo contra cada actividad principal en evaluación. Luego la calificación dada se multiplicó dos veces, es decir, una vez por el porcentaje asignado a la actividad principal y la otra vez por el porcentaje asignado a la actividad de apoyo, para luego sumar el resultado de las dos multiplicaciones y obtener la calificación definitiva.

Cabe aclarar que en las calificaciones que se realizaran en toda la matriz podían repetirse los números de la escala de uno a cinco, porque podían haber varias calificaciones con el mismo nivel de interpretación de la importancia para ventaja competitiva. Después de haber calificado cada actividad de apoyo contra cada actividad principal se procedió a sumar los resultados de las calificaciones para obtener la suma total o simplemente el "total" de las calificaciones por cada actividad. Por lo tanto, en la Tabla 3 y la Tabla 4 se pueden observar los rangos de interpretación del total para las actividades principales y para las actividades de apoyo respectivamente. Los intervalos que componen cada uno de los rangos se han obtenido al simular escenarios en los que todas las calificaciones llegaban a ser cinco o cuatro o tres o dos o uno. Los totales que obtenía cada actividad también permitían identificar sus ventajas competitivas (junto con fortalezas) y debilidades de manera general. Inclusive, estos totales pueden permitir la identificación del nivel de margen o creación de valor que se esté generando en la cadena de valor, teniendo en cuenta el concepto de valor de Porter que se sintetiza en obtener ganancia sobre sus costos, es decir que sus ingresos menos costosos resulten en utilidades.

Tabla 3. Rango de interpretación del total para actividad principal

\begin{tabular}{|c|c|c|c|}
\hline NÚMERO & RANGO & COLOR & INTERPRETACIÓN \\
\hline $\mathbf{5}$ & 3,53 a 4,4 & Verde & Ventaja competitiva \\
\hline $\mathbf{4}$ & 2,65 a 3,52 & Azul & Potencial de ventaja competitiva \\
\hline $\mathbf{3}$ & 1,77 a 2,64 & Amarillo & Regular \\
\hline $\mathbf{2}$ & 0,89 a 1,76 & Naranja & Debilidad menor \\
\hline $\mathbf{1}$ & 0,00 a 0,88 & Rojo & Debilidad mayor \\
\hline
\end{tabular}

Fuente: elaboración propia

Tabla 4. Rango de interpretación del total por actividad de apoyo

\begin{tabular}{|c|c|c|c|}
\hline NÚMERO & RANGO & COLOR & INTERPRETACIÓN \\
\hline $\mathbf{5}$ & 4,5 a 5,5 & Verde & Ventaja competitiva \\
\hline $\mathbf{4}$ & 3,4 a 4,4 & Azul & Potencial de ventaja competitiva \\
\hline $\mathbf{3}$ & 2,3 a 3,3 & Amarillo & Regular \\
\hline $\mathbf{2}$ & 1,2 a 2,2 & Naranja & Debilidad menor \\
\hline $\mathbf{1}$ & 0,0 a 1,1 & Rojo & Debilidad mayor \\
\hline
\end{tabular}

Fuente: elaboración propia 


\section{DISCUSIÓN}

La cadena de valor como herramienta gerencial ayuda a analizar y diagnosticar fuentes de ventaja competitiva que representan diferenciación para las organizaciones en sus actividades primarias y actividades de apoyo, enfocándose en la mayor generación de valor. Por lo tanto, al aplicar el análisis y la evaluación de la cadena de valor se debería saber dónde disminuir costos y dónde potenciar u optimizar ganancias, incrementando favorablemente el nivel de comunicación y promoviendo la transferencia de tecnología y la generación de una gobernanza más adecuada (Peña et al., 2008).

A partir de la metodología propuesta se da un mayor alcance, analizando cualitativa y cuantitativamente la relación de cada actividad principal con cada actividad de apoyo. De esta manera, las descripciones de relaciones entre actividades principales y de apoyo, la doble multiplicación, los rangos y la suma total de las calificaciones constituyen las principales diferencias de esta evaluación interna para identificar ventajas competitivas y debilidades frente a los modelos propuestos en la metodología de evaluación a partir de factores de integración, formulada por De la Hoz et al. (2011), la metodología de evaluación a partir de FODA, la metodología de evaluación a partir de la ponderación de actividades por categorías, según Arce y Calves (2008), y la metodología de evaluación y priorización de cadenas de valor agrícolas para la responsabilidad social empresarial, según Scott (2014).

\section{CONCLUSION}

Reconocida la importancia de la cadena de valor como instrumento de diagnóstico empresarial y por el valor que crea en las organizaciones, se decidió el diseño de una metodología para llevar a cabo la evaluación interna tanto de manera cuantitativa como cualitativa de la cadena de valor según el modelo teórico de Porter. Esta permite identificar actividades principales y actividades de apoyo fuertes como las ventajas de competitividad, y por otro lado las necesidades de mejora catalogadas como falencias o debilidades, así como las relaciones entre ellas, mejor conocidas como eslabones que influencian la dinámica de la cadena de valor.

Por lo tanto, como contribución a futuras investigaciones y aplicaciones empresariales bien sea a una empresa o sector económico, la evaluación es un buen complemento al diseño de la cadena de valor, puesto que permite una perspectiva más crítica y detallada sobre la misma y aporta a una buena base de información y un componente de evaluación numérico para priorizar la toma de decisiones. De este modo se puede ser más objetivo, más allá de las descripciones cualitativas realizadas. Adicionalmente, a través de esta calificación se identifican eslabones que conlleven la generación de valor representada en ventajas competitivas específicas entre las actividades. Por otra parte, también se pueden identificar debilidades concretas entre las actividades que requieran la implementación de planes de mejora y control de estos.

Así mismo, al identificar ventajas competitivas y factores de mejora se obtiene una percepción sobre el nivel o grado de competitividad y productividad de la organización y/o sector, sobre los generadores de valor y sobre las actividades de la cadena que se deben potenciar. De esta manera se habrán evaluado todas las actividades o áreas de una empresa o sector económico que estén generando valor o lo estén afectando. La evaluación aplicada se convierte en una herramienta gerencial para orientar la toma de decisiones para aumentar la competitividad y la efectividad en las organizaciones. Un complemento a la metodología de evaluación interna propuesta es también desarrollar y aplicar una metodología de evaluación externa de las variables que afectan el entorno de una cadena de valor de una organización y que por ende en una buena parte de los casos se encuentran fuera del rango de control, pero representan oportunidades $o$ amenazas.

\section{Declaración sobre conflictos de interés}

Los autores del presente artículo de investigación declaran que son independientes y no existe ningún conflicto de interés en el desarrollo de la investigación ni en esta publicación. El presente artículo nace del proyecto de investigación "Generación de valor para la competitividad con perspectiva de internacionalización de la cadena de valor de cacao de Norte de Santander en el escenario de la paz y el postconflicto", financiado por Colciencias, ahora Ministerio de Ciencia, Tecnología e Innovación de Colombia, en convenio con la Universidad Francisco de Paula Santander dentro del programa de Jóvenes Investigadores Convocatoria Colciencias 812 de 2018.

\section{REFERENCIAS BIBLIOGRÁFICAS}

Arce, B. y Calves, S. (2008). La evaluación de las cadenas de valor como estrategia para la competitividad de las Pymes. Revista electrónica de ciencias sociales Tecsistecatl, 5. https://www.eumed.net/rev/tecsistecatl/n 
METODOLOGÍA PARA LA EVALUACIÓN INTERNA DE UNA CADENA DE VALOR

\section{5/acch.pdf}

Boehlje, M. (1999). Structural changes in the agricultural industries: How do we measure, analyze, and understand them? American Journal of Agricultural Economics, $\quad$ 81, 1028-1041. https://www.jstor.org/stable/1244080

Chávez, J. (2012). Cadena de valor, estrategias genéricas y competitividad: el caso de los productores de café orgánico del municipio de Tanetze de Zaragoza, Oaxaca [tesis de maestría, Instituto Tecnológico de Oaxaca]. Repositorio Instituto Tecnológico de Oaxaca. http://www.eumed.net/librosgratis/2013b/1345/tipoinvestigacion.html

Craig, C. y Douglas, S. (1997). Managing the Transnational Value Chain: Strategies for Firms from Emerging Markets. Journal of International Marketing, 5(3), 71-84.

http://www.jstor.org/stable/25048694

De la Hoz, E., Fontalvo, T. y Morelos, J. (2011). Modelo de evaluación de cadenas de suministro en el sector de confecciones de Barranquilla. Revista INGENIARE Universidad Libre Barranquilla, 6 (11), 79-92. https://doi.org/10.18041/19092458/ingeniare.11.647

Devoto, R. (2011). Ventaja Competitiva y Cadena de Valor. Pontificia Universidad Católica de Valparaíso. Valparaíso,
Chile.

http://www.pucv.cl/uuaa/site/artic/2018 1123/asocfile/20181123195329/apunted ocenteventajacompettivaycadenadevalor rd.pdf

Hergert, M. y Morris, D. (1989). Accounting Data for Value Chain Analysis. Strategic Management Journal, 10(2), 175-188. http://www.jstor.org/stable/2486509

Higgins, A., Miller, C., Archer, A., Ton, T., Fletcher, C. y McAllister, R. (2010). Challenges of operations research practice in agricultural value chains. The Journal of the Operational Research Society, 61(6), 964-973. http://www.jstor.org/stable/40608268

Peña, Y., Nieto, P. y Díaz, F. (2008). Cadenas de valor: un enfoque para las agrocadenas. Equidad y Desarrollo, 9, 77-85. http://dx.doi.org/10.19052/ed.279

Pérez, O. y Calves, S (2008). Ponderación de las cadenas de valor. Curso a empresarios de la UNECA. Cuba.

Perona, M. y Miragliotta G. (2004). Complexity management and supply chain performance assessment. A field study and conceptual framework. International Journal of Production Economics, 90, 103-115. https://doi.org/10.1016/S09255273(02)00482-6

Piana, C., Featherstone, A. y Boland, M. (2005). Vertical Integration in Ecuador: The Case of Fresh-Cut Pineapples. Review of 
Agricultural Economics, 27(4), 593-603.

http://www.jstor.org/stable/3700769

Porter, M. (1985). Ventaja competitiva:

Creación y sostenimiento de un desempeño superior. Free Press.

Porter, M. (2006). Ventaja competitiva. CECSA.

Quintero, J. y Sánchez, J. (2006). La cadena de valor: Una herramienta del pensamiento estratégico. Revista Telos, 8, 377-389. http://www.redalyc.org/articulo.oa?id=9 9318788001

Scott, G. (2014). Agregando valores a las cadenas de valor. Revista de Administração de Empresas, 54, 67-79. http://www.redalyc.org/articulo.oa?id=1 55129752007

Shapiro, J., Singhal, V. y Wagner, S. (1993). Optimizing the Value
Chain. Interfaces, 23(2),

102-117. http://www.jstor.org/stable/25061732

Valentine, I. (2005). An emerging model of a systems agriculturalist. System Research and Behavioral Science, 22, 109-118. https://doi.org/10.1002/sres.678

Vásquez, E. y Bastos, L. (2018). Análisis de metodologías para la evaluación de cadenas de valor: caso del aguacate en Norte de Santander. Revista Respuestas, 23(1), 66-69. https://revistas.ufps.edu.co/index.php/re spuestas/article/view/1504

Wright, P. (1987). A Refinement of Porter's Strategies. Strategic Management Journal, $\quad$ 8(1), 93-101. http://www.jstor.org/stable/2486148 